\title{
Is metabolic syndrome the link between obesity and postmenopausal breast cancer?
}

\author{
L. A. Healy ${ }^{2}$, A. M. Ryan ${ }^{2}$, P. Carroll ${ }^{1}$, D. Ennis ${ }^{1}$, T. Boyle ${ }^{1}$, M. J. Kennedy ${ }^{3}$, E. Connolly ${ }^{1}$, \\ and J. V. Reynolds ${ }^{1}$ \\ Department of Surgery ${ }^{1}$, Department of Clinical Nutrition ${ }^{2}$, Academic Unit of Clinical and Medical Oncology ${ }^{3}$, \\ St James's Hospital and Trinity College Dublin
}

Obesity is implicated both in breast cancer risk and cancer mortality. The proposed mechanisms underpinning these associations are abdominal obesity, insulin resistance, altered sex steroids, adipokines, inflammation and oxidative stresses. The clustering of some of these risk factors is central to the definition of metabolic syndrome (MetS), and the relationship between MetS and breast cancer biology is unknown ${ }^{(1)}$.

The aim of this prospective observational study of postmenopausal breast cancer patients was to examine the incidence of MetS and any association with tumour pathology. Individuals underwent a metabolic and nutritional assessment prior to treatment. MetS was defined according to the International Diabetes Federation definition ${ }^{(2)}$.

One hundred and seven female breast cancer patients were consented. The median age was 67 years (range 40-84) and mean BMI was $28.3 \pm 5.3 \mathrm{~kg} / \mathrm{m}^{2}$, with $87 \%$ centrally obese. MetS was diagnosed in $40 \%$ patients, almost double the predicted Irish level ${ }^{(3)}$. MetS was significantly associated with central obesity $(P<0.005)$; a $13 \mathrm{~cm}$ greater waistline, higher total and trunk fat mass, as well as higher systolic BP $(P=0.008)$, and insulin levels $(P=0.015)$. Obesity was associated with larger tumours $(P=0.021)$, while MetS was significantly associated with a later stage of disease $(P<0.001)$ and axillary node involvement $(P=0.028)$.

MetS and central obesity are common in Irish postmenopausal breast cancer patients. MetS appears to be associated with an aggressive tumour biology, and this may have significance in preventive and novel treatment approaches.

1. Alberti KGMM et al. (2006) Diabet Med 23, 469-480.

2. Cowey S \& Hardy RW (2006) Am J Pathol 168, 1505-1522.

3. Waterstone DF et al. (2009) Ir J Med Sci 178(2), 161-6. 